Ann. Biol. anim. Bioch. Biophys., I969, 9 (3), 44I-443.

\title{
ACGÉLÉRATION DU RYTHME DE LA REPRODUCTION CHEZ LE MOUFLON DE CORSE (OVIS AMMON MUSIMON SCHREBER, 1782) AU MOYEN DE CYCLES PHOTOPÉRIODIQUES SEMESTRIELS
}

\author{
J. ROUGEOT \\ Station centrale de Physiologie animale, \\ Centre national de Recherches zootechniques, 78 -Jouy-en-Josas \\ Institut national de la Recherche agronomique
}

On sait que des Brebis, soumises à des cycles photopériodiques de 6 mois ayant une amplitude de la variation de la photopériode comprise entre un minimum de 8 heures et un maximum de 16 heures par 24 heures, présentent deux saisons d'activité sexuelle par an, limitées aux phases de jours croissants (ROUGEOT, I96I ; MAULÉON et RoUGEOT, 1962). Nous avons répété la même expérience sur des Mouflons de Corse qui, en conditions naturelles de photopériodisme et, comme l'ont montré des observations faites dans la nature (PFEFFER, I967) aussi bien que dans notre élevage, n'ont strictement qu'une saison d'activité sexuelle par an s'étendant sur les mois de novembre et de décembre, c'est-à-dire en jours courts sous photopériode décroissante. Mais, cette fois-ci, nous avons, en plus, laissé les animaux se reproduire afin d'observer comment pouvaient se succéder les mises bas, étant donné la période de 6 mois des cycles photopériodiques, la durée de 5 mois de la gestation et l'interférence possible entre l'anœstrus post partum et l'anœstrus saisonnier (MaUlÉon et DAUZIER, I965) qui, dans ces conditions, s'étend sur toute la phase des jours décroissants chez la Brebis.

A cet effet, un mâle et une femelle ont été soumis à des cycles photopériodiques semestriels du 21 décembre i 964 au 21 décembre ig68: les animaux libres de sortir dans un enclos situé à l'extérieur pendant la journée, étaient enfermés le soir à 16 hevres jusqu'au lendemain matin à 9 heures dans une cellule étanche à la lumière du jour, où ils recevaient un complément de lumière iourni par des tubes fluorescents dont le fonctionnement était réglé par des interrupteurs horaires.

Comme chez la Brebis, on a obtenu pour la femelle deux saisons d'activité sexuelle par an, limitées aux phases de jours croissants (fig. I) : les saillies, qui ont eu lieu pendant ces phases, ont été fécondes et les jeunes obtenus, sauf celui du cycle $n^{\circ} 6$ mort à la naissance par accident, ont tous été normalement élevés et sevrés à trois mois.

La durée de l'anœstrus post-partum a pu être déterminée dans les cycles où il se termine pendant la saison d'activité sexuelle des jours croissants : elle est de $43,58,43,44$ et 45 jours aux cycles numéros $2,5,6,7$ et ro respectivement. L'intervalle entre deux saillies ne peut donc être inférieur à 6,5 mois et il en résulte un décalage progressif de la date d'agnelage par rapport aux cycles photopériodiques de 6 mois : cela entraîne une réduction progressive de la saison d'activité sexuelle possible qui s'annule lorsque la:mise bas a lieu tardivement en jours croissants, la fin de l'anostrus 

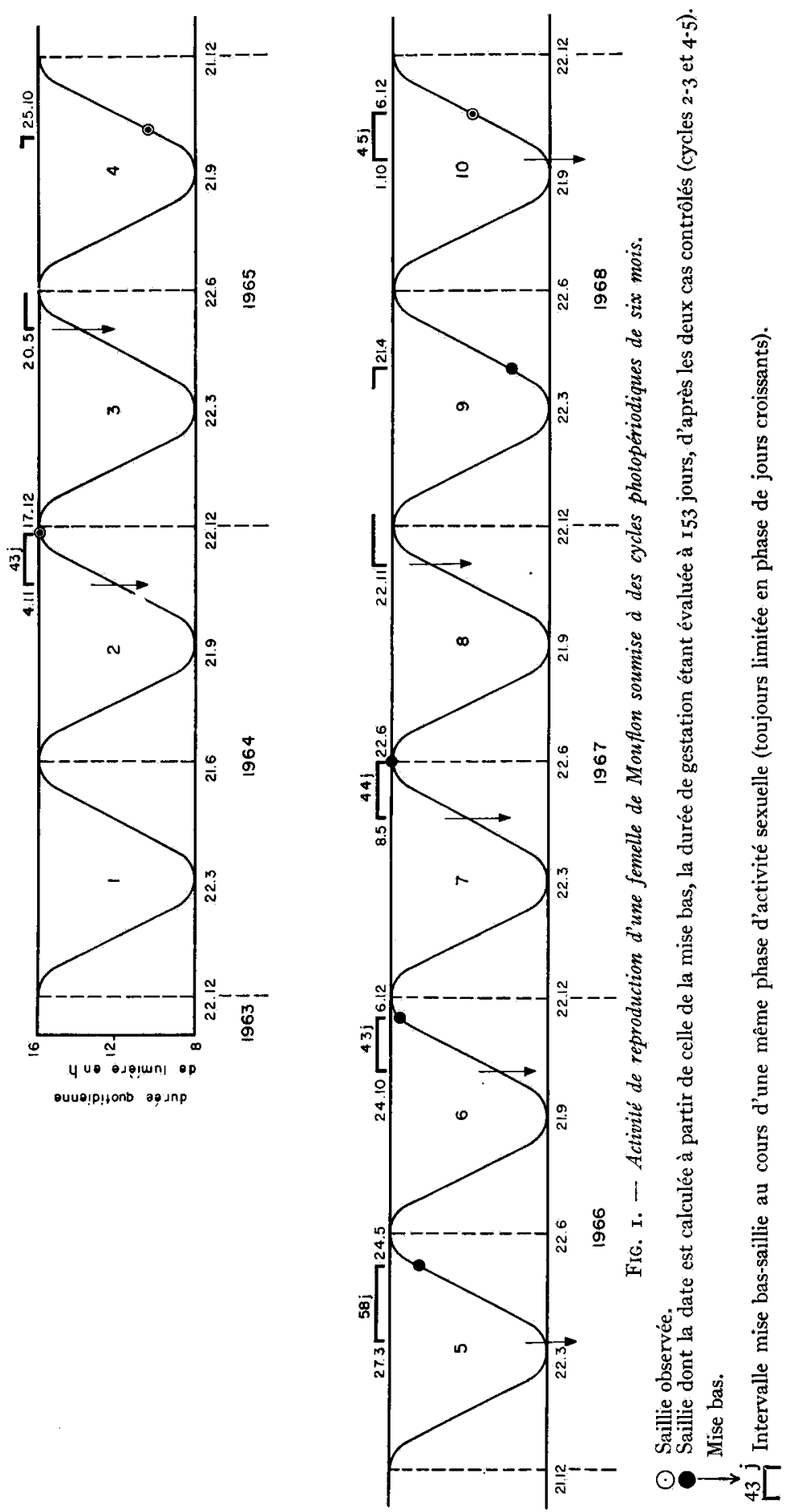
post partum venant en coïncidence avec le début de l'anœestrus saisonnier des jours décroissants (cycles numéros 3 et 8).

Ainsi, cette expérience confirme les observations faites chez la Brebis en soulignant encore mieux la contradiction qui existe entre l'action du cycle photopériodique annuel naturel où l'activité sexuelle n'a lieu qu'en début de période de jours courts sous photopériode décroissante et celle du cycle photopériodique semestriel qui entraîne une activité sexuelle exclusivement en jours croissants. Aucune explication physiologique satisfaisante ne peut être encore proposée.

Sur le plan pratique, cette expérience montre la possibilité d'obtenir chez les Ovins, par un conditionnement lumineux très simple, presque deux agnelages par an. On peut même penser qu'en sevrant précocement les agneaux, il serait possible de réduire l'anœestrus de lactation à une trentaine de jours (MAULÉon et DAUZIER, 1965) et de conserver un rythme rigoureux d'agnelage tous les six mois.

Reçu pour publication en avril 1969.

\title{
SUMMARY
}

\begin{abstract}
EFFECT OF 6 MONTH PHOTOPERIOD CYCLES ON REPRODUCTIVE EFFICIENCY
\end{abstract}
IN THE CORSICAN MOUFLON (" OVIS AMMON MUSIMON SCHREBER, I 782)

A pair of Mouflons was submitted to 6 month cycles of 8 to 16 hours of daily photoperiod. The female exhibited two sexual seasons per year, under increasing daylength, versus one sexual season under decreasing daylength in natural lighting conditions. This result further substantiates our previous investigations on the Ewe.

The female was mated and pregnancies were followed by normal lambings. The average duration of post-partum anoestrus was $43-45$ days, and the shortest time interval between matings was 6.5 months. This resulted in an increasing lag of the reproductive cycle behind the photoperiod cycle; when the post-partum anostrus came to end under decreasing daylength, the next mating was delayed untill the end of the seasonal anoestrus (graph I.). tion.

Almost two yearly lambings can therefore be obtained by a simple photoperiodic manipula-

\section{RÉFÉRENCES BIBLIOGRAPHIQUES}

Mauléon P., Rougeot J., I962. Régulation des saisons sexuelles chez les Brebis de races différentes au moyen de divers rythmes lumineux. Ann. Biol. anim. Bioch. Biophys, , 2, 209-222.

Mauléon P., Dauzier L., I965. Variation de la durée de l'ancestrus de lactation chez les Brebis de race Ile-de-France. Ann. Biol, anim. Bioch. Biophys., 5, I3 $3^{\mathrm{I}-\mathrm{I} 43 .}$

PFEFFer P., I 967 . Le Mouflon de Corse (Ovis ammon musimon Schreber, 1782). Position systématique, écologie et éthologie comparées. Mammalia, 31 (supplément), I=25I.

Rougeot J., I96r. Actions comparées des variations périodiques annuelles et semestrielles de la durée quotidienne de l'éclairement sur les cycles des follicules des jarres courts de la toison des Brebis Limousines. Relation avec leur cycle de reproduction. Ann. Biol, anim. Bioch. Biophys., 1, 385-402. 